\title{
Euler's Totient Function, the Mangoldt Function, and a Sequence of Mertens Function Values
}

\author{
Darrell Cox ${ }^{1}$, Sourangshu Ghosh ${ }^{2}$ and Eldar Sultanow ${ }^{3}$ \\ ${ }^{1}$ Department of Mathematics, Grayson County College, United \\ States \\ ${ }^{2}$ Department of Civil Engineering,Indian Institute of Technology \\ Kharagpur, India \\ ${ }^{3}$ Potsdam University, Chair of Business Informatics, Processes and \\ Systems, Potsdam, Germany
}

\begin{abstract}
The Möbius function is commonly used to define Euler's totient function and the Mangoldt function. Similarly, the summatory Möbius function (the Mertens function) can be used to define the summatory totient function and the summatory Mangoldt function (the second Chebyshev function).
\end{abstract}

\section{Introduction}

If $n \geq 1$ the Euler totient function $\varphi(n)$ is defined to be the number of positive integers not exceeding $n$ which are relatively prime to $n$. If $n>1$, then $n=p_{1}^{a_{1}} p_{2}^{a_{2}} \cdots p_{k}^{a_{k}}$ where $p_{1}, p_{2}, \ldots, p_{k}$ are primes. The Möbius function $\mu(n)$ is defined as follows: $\mu(1)=1, \mu(n)=(-1)^{k}$ if $a_{1}=a_{2}=\cdots=a_{k}=1$, or $\mu(n)=0$ otherwise. Note that $\mu(n)=0$ if and only if $n$ has a square factor $>1$. The Euler totient function is related to the Möbius function through the following formula:

(1) $\varphi(n)=\sum_{d \mid n} \mu(d) \frac{n}{d}$

See Theorem 2.3 of Apostol's [1] book. The Mangoldt function $\Lambda(n)$ is defined to be $\log (p)$ if $n=p^{m}$ for some prime $p$ and some $m \geq 1$, or 0 otherwise. $\Lambda(n)$ is expressed in terms of the logarithm as follows:

(2) $\Lambda(n)=\sum_{d \mid n} \mu(d) \log \frac{n}{d}=-\sum_{d \mid n} \mu(d) \log (d)$

See Theorem 2.11 of Apostol's book. The second Chebyshev function $\psi(x)$ is defined to be $\sum_{n \leq x} \Lambda(n)$. The average order of $\varphi(n)$ is: 
(3) $\sum_{n \leq x} \varphi(n)=\frac{3}{\pi^{2}} x^{2}+O(x \log x)$ so the average order of $\varphi(n)$ is $3 n / \pi^{2}$.

See Theorem 3.7 of Apostol's book. Let $\Phi(x)$ denote $\sum_{n=1}^{x} \varphi(n)$. Let $M(x)$ denote $\sum_{n=1}^{x} \mu(n)$.

\section{The Redheffer Matrix}

The squarefree divisors of $n=2,3,4,5,6, \ldots$ are $2,3,2,5,(2,3,6), 7,2,3$, $(2,5,10), 11,(2,3,6), \ldots$ For example, for $n \leq 12$ there are $\lfloor 12 / 2\rfloor 2$ 's, $\lfloor 12 / 3\rfloor$

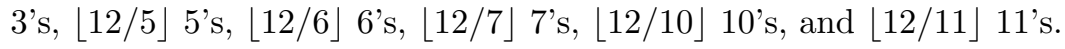

Mikolás [2] proved that $\sum_{i=1}^{x} M(\lfloor x / i\rfloor)=1$. In general, $\sum_{i=1}^{x} M(\lfloor x /($ in $)\rfloor)=1$, $n=1,2,3, \ldots, x$ (since $\lfloor\lfloor x / n\rfloor / i\rfloor=\lfloor x /(i n)\rfloor)$. Let $R_{x}^{\prime}$ denote a square matrix where element $(i, j)$ equals 1 if $j$ divides $i$ or 0 otherwise. (In a Redheffer matrix, element $(i, j)$ equals 1 if $i$ divides $j$ or if $j=1$. Redheffer [3] proved that the determinant of such a $x$ by $x$ matrix equals $M(x)$.) Let $T_{x}$ denote the matrix obtained from $R_{x}^{\prime}$ by element-by-element multiplication of the columns by $M(\lfloor x / 1\rfloor), M(\lfloor x / 2\rfloor), M(\lfloor x / 3\rfloor), \ldots, M(\lfloor x / x\rfloor)$. For example, the $T_{x}$ matrix for $x=12$ is

$\begin{array}{rrrrrrrrrrrr}-2 & 0 & 0 & 0 & 0 & 0 & 0 & 0 & 0 & 0 & 0 & 0 \\ -1 & -1 & 0 & 0 & 0 & 0 & 0 & 0 & 0 & 0 & 0 & 0 \\ -1 & 0 & -1 & 0 & 0 & 0 & 0 & 0 & 0 & 0 & 0 & 0 \\ -1 & -1 & 0 & -1 & 0 & 0 & 0 & 0 & 0 & 0 & 0 & 0 \\ 0 & 0 & 0 & 0 & 0 & 0 & 0 & 0 & 0 & 0 & 0 & 0 \\ 0 & 0 & 0 & 0 & 0 & 0 & 0 & 0 & 0 & 0 & 0 & 0 \\ 1 & 0 & 0 & 0 & 0 & 0 & 1 & 0 & 0 & 0 & 0 & 0 \\ 1 & 1 & 0 & 1 & 0 & 0 & 0 & 1 & 0 & 0 & 0 & 0 \\ 1 & 0 & 1 & 0 & 0 & 0 & 0 & 0 & 1 & 0 & 0 & 0 \\ 1 & 1 & 0 & 0 & 1 & 0 & 0 & 0 & 0 & 1 & 0 & 0 \\ 1 & 0 & 0 & 0 & 0 & 0 & 0 & 0 & 0 & 0 & 1 & 0 \\ 1 & 1 & 1 & 1 & 0 & 1 & 0 & 0 & 0 & 0 & 0 & 1\end{array}$

Let $U_{x}$ denote the matrix obtained from $T_{x}$ by element-by-element multiplication of the columns by $\varphi(j)$. The sum of the sums of the columns of $U_{x}$ then equals $\Phi(x) . i=\sum_{d \mid i} \varphi(d)$, so $\sum_{i=1}^{x} M(\lfloor x / i\rfloor) i$ (the sum of the sums of the rows of $U_{x}$ ) equals $\Phi(x)$. This relationship was proved by Cox [4] (and previously by Mikolás).

(4) $\sum_{i=1}^{x} M(\lfloor x / i\rfloor) i=\Phi(x)$

A plot of the sums of the rows for $x=8000$ is given in Figure 1 . 


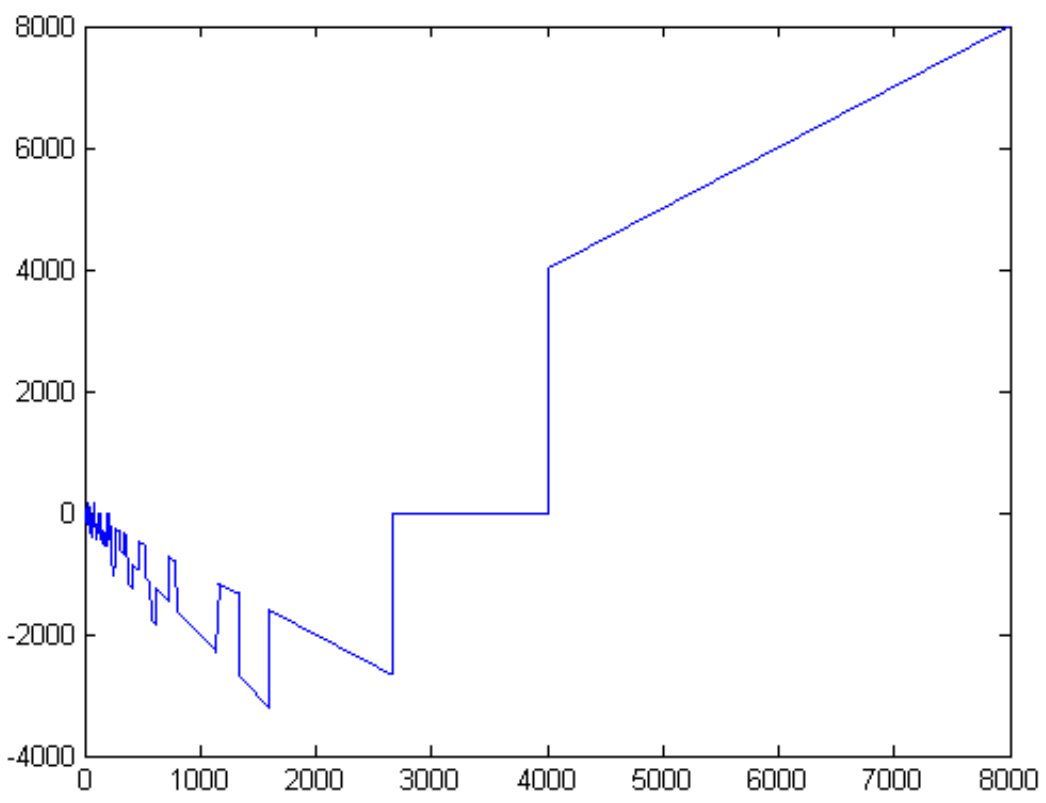

Figure 1: Plot of the sums of the rows for $x=8000$

This plot indicates that $\Phi(x)$ equals a series involving the squarefree numbers $1,2,3,5,6,7,10, \ldots$ For $x=200$, the values from $\lfloor x / 2\rfloor+1$ to $\lfloor x / 1\rfloor$ are 101 , $102,103, \ldots, 200$ (the initial value is $\lfloor x / 2\rfloor+1$ ). The values from $\lfloor x / 3\rfloor+1$ to $\lfloor x / 2\rfloor$ are 0 . The values from $\lfloor x / 5\rfloor+1$ to $\lfloor x / 3\rfloor$ are $-41,-42,-43, \ldots-66$. The values from $\lfloor x / 6\rfloor+1$ to $\lfloor x / 5\rfloor$ are $-68,-70,-80, \ldots-66$ (the initial value is the negative of twice $\lfloor x / 6\rfloor+1$ ). The process continues with larger squarefree divisors. Several squarefree divisors may map to the same term in the series the smallest is selected. There are other multiples of the initial values and other multiples of 0 can occur. For $x=200$, the squarefree numbers and corresponding multiples are $(2,1),(3,0),(5,-1),(6,-2),(7,-1),(10,-2),(11,-1)$, $(13,-2),(14,-3),(15,-2),(17,-1),(19,-2),(21,-3),(23,-1),(26,-2)$, $(29,-1),(34,-3),(41,0),(51,-3),(67,-1),(101,1)$, and $(201,-8)$. The multiple for a squarefree number $q$ is $M(q-1)$. Each term in the series is the sum of an arithmetic progression and is thus quadratic in terms of $x$. About 0.81 times the first term equals $\Phi(x)$.

If $q<\sqrt{x},\lfloor x / q\rfloor \neq\lfloor x / r\rfloor$ where $r$ is the next squarefree number. The asymptotic density of the squarefree numbers is $\frac{6}{\pi^{2}}$. (Even for $x$ as small as 10, this gives 6.08. The actual number is 6 . For $x<50$, this gives 30.40. The actual number is 30.) The sum of the terms corresponding to the first $\left\lceil\sqrt{\frac{6 x}{\pi^{2}}}\right\rceil$ squarefree numbers is an approximation of $\Phi(x)$ typically accurate to less than $0.5 \%$ relative error for fairly large $x$ values (say greater than 300 ). Properties of the 
Mertens function can be used to give a better approximation.

\section{Properties of the Sequence $M(\lfloor x / 1\rfloor), M(\lfloor x / 2\rfloor)$, $M(\lfloor x / 3\rfloor), \ldots, M(\lfloor x / x\rfloor)$}

A plot of the largest $i$ value, $i<\lfloor x / 3\rfloor+1$, where $M(\lfloor x / i\rfloor)$ is greater than or equal to zero for $x=200,400,600, \ldots, 10000$ is given in Figure 2 .

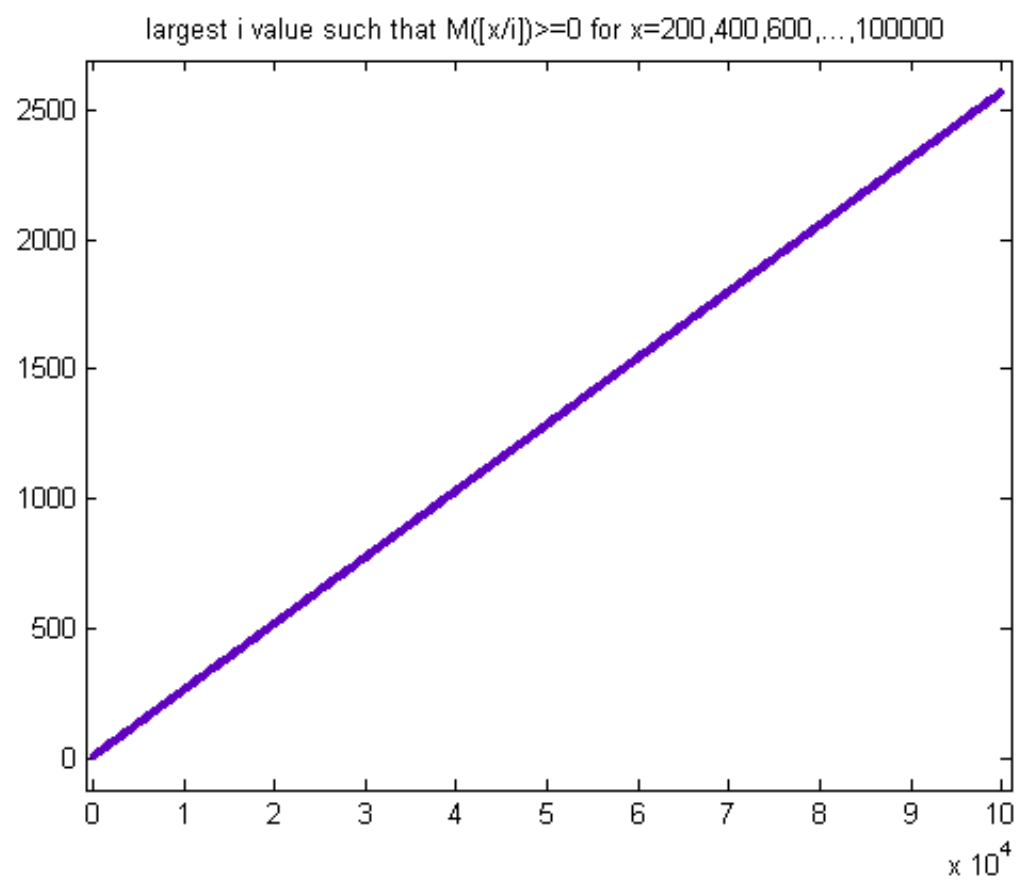

Figure 2: Plot of largest $i$ value, $i<\lfloor x / 3\rfloor+1$, such $M(\lfloor x / i\rfloor) \geq 0$

The $i$ value for $x=200$ is 5 . The $i$ values increase by 5 up to $x=1400$ and then increase by 6 (so that the $i$ value for $x=1600$ is 41). The $i$ values then increase by 5 up to $x=3000$ and then increase by 6 (so that the $i$ value for $x=3200$ is 82). There are either 7 or 8 elements in these groups of $i$ values that increase by 5 . The increase from one group to the next is always 6 . The numbers of elements in the first five groups are $7,8,8,8$, and 7 . The numbers of elements in the subsequent five groups are $8,8,8,8$, and 7 .

The $i$ value for $x=2000$ is 51. A plot of the sums of the rows of $U_{x}$ starting with row \#52 for $x=2000$ is given in Figure 3 . 


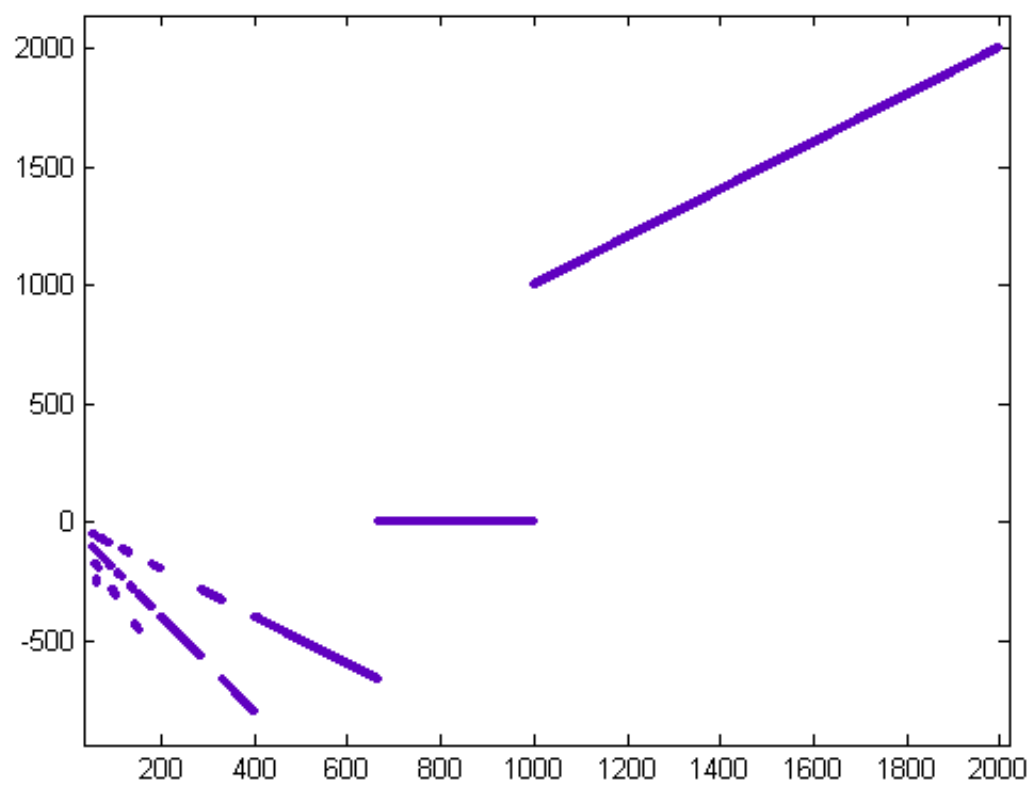

Figure 3: Plot of the sum of rows for $x=2000$

Excluding the first 51 sums of rows gives orderly results - there is one line with a slope of 1 , one line with a slope of 0 , and four intermittent lines with slopes of $-1,-2,-3$, and -4 . A plot of this approximation of $\Phi(x)$ and $\Phi(x)$ for $x=200$, $400,600, \ldots, 2000$ is given in Figure 4. 


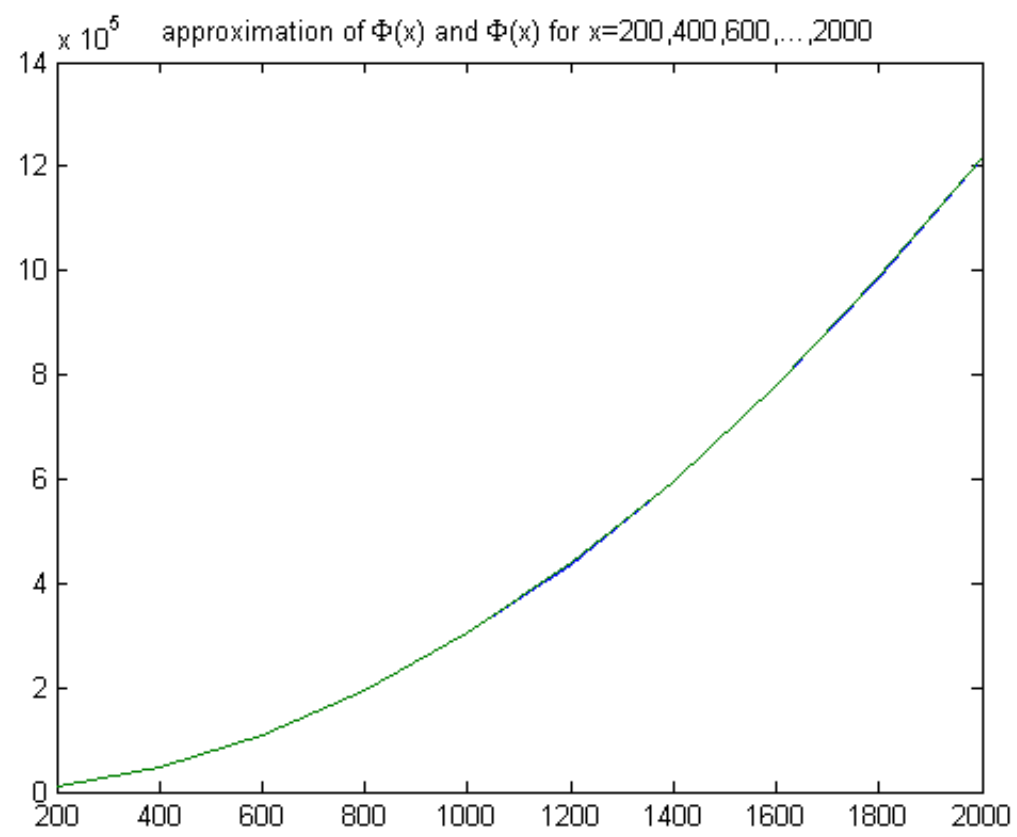

Figure 4: Plot of approximation of $\Phi(x)$ and $\Phi(x)$

A plot of the quadratic least-squares fit of the approximation of $\Phi(x)$ is given in Figure 5. 


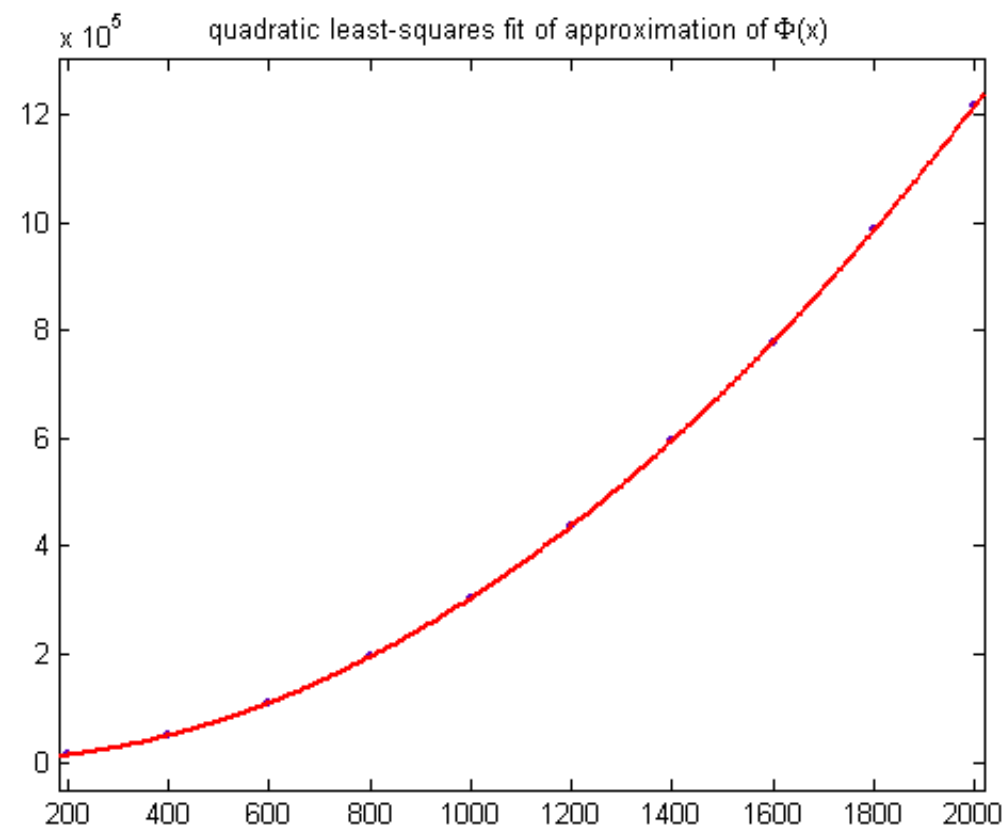

Figure 5: Plot of quadratic least-squares fit of approximation of $\Phi(x)$

A plot of the quadratic least-squares fit of the approximation of $\Phi(x)$ minus $\Phi(x)$ is given in Figure 6. 


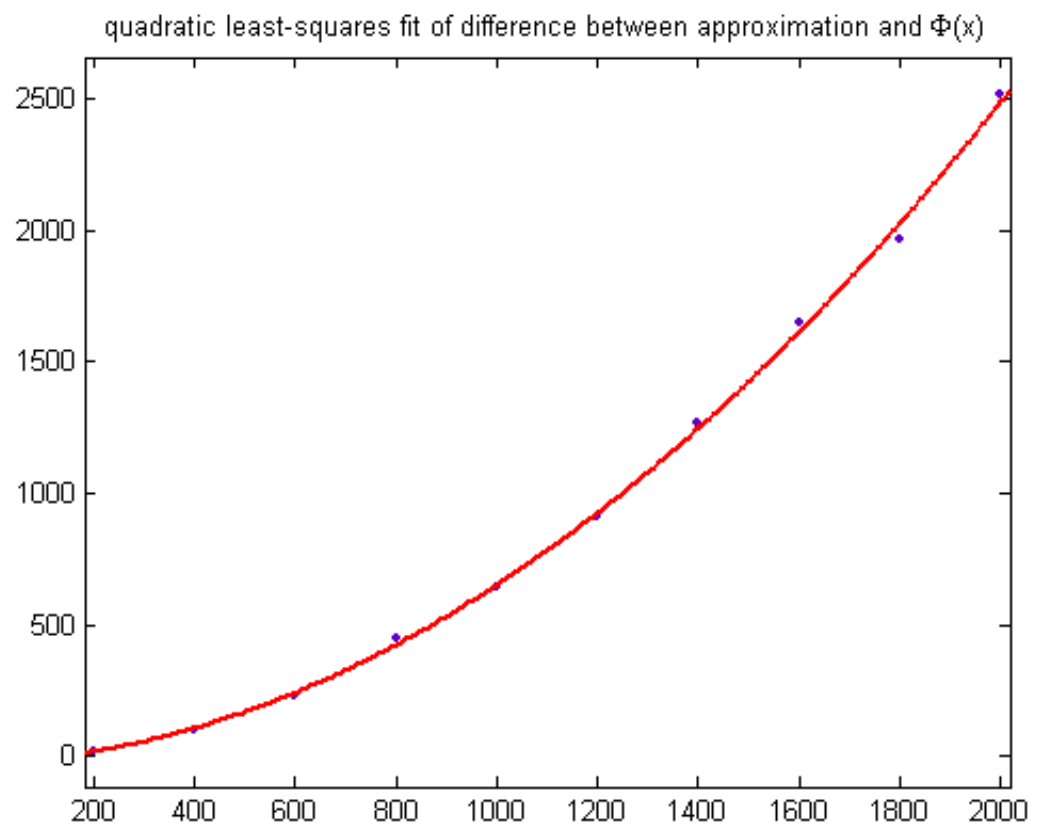

Figure 6: Plot of quadratic least-squares fit of difference of approximation of $\Phi(x)$ and $\Phi(x)$

A plot of the largest $i$ values for $x$ up to 200 is given in Figure 7 . 


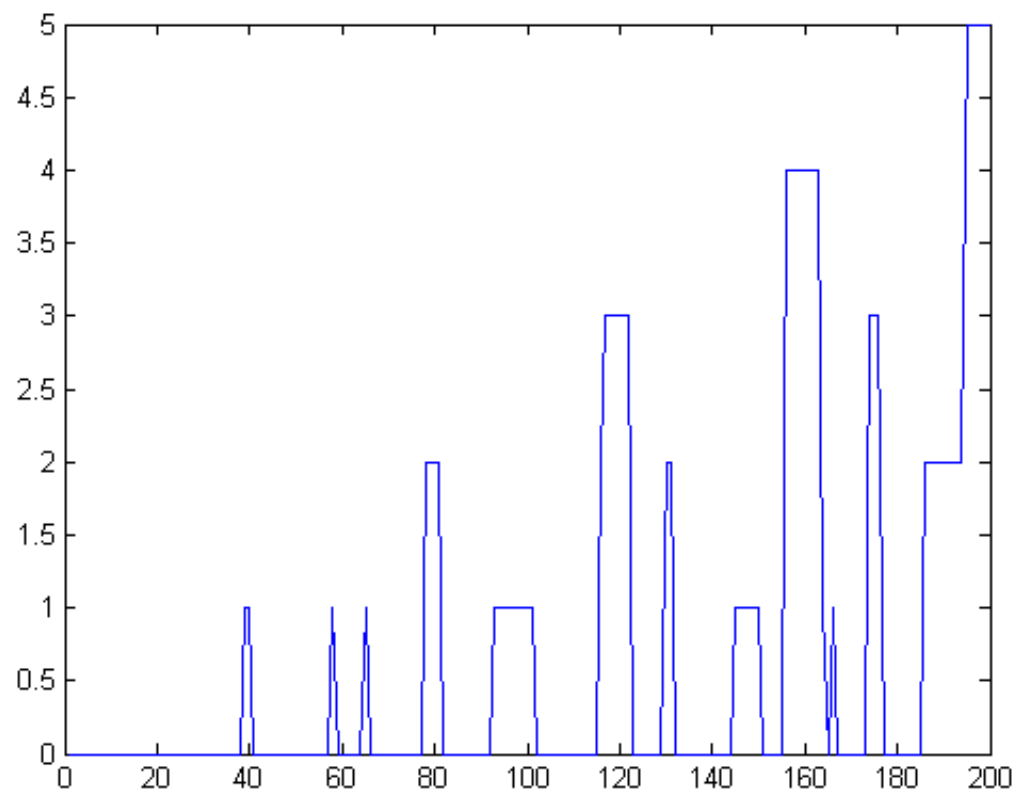

Figure 7: Plot of largest $i$ values up to $x=200$

The largest $i$ values increase more systematically after $x=800$. A plot of the largest $i$ values for $x=801$ to 1000 is given in Figure 8 . 


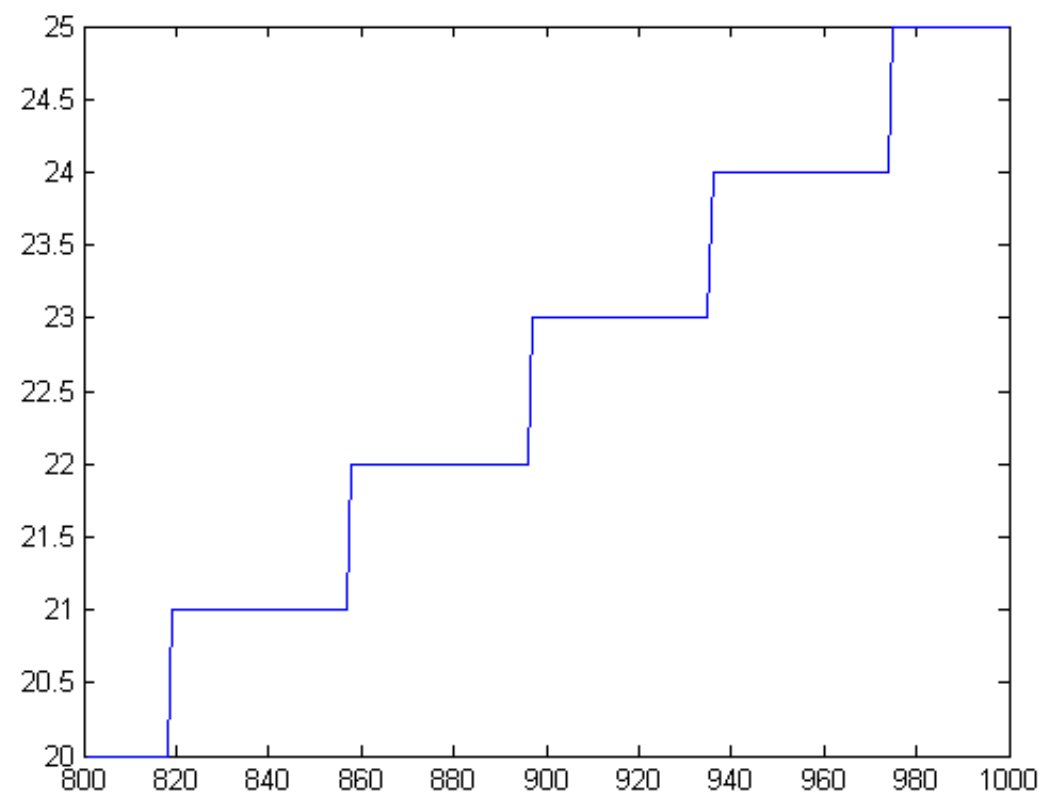

Figure 8: Plot of largest $i$ values for $x=801$ to 1000

A plot of the sum of the positive $M(\lfloor x / i\rfloor)$ values up to the largest $i$ value for $x=200,400,600, \ldots, 100000$ is given in Figure 9 . 


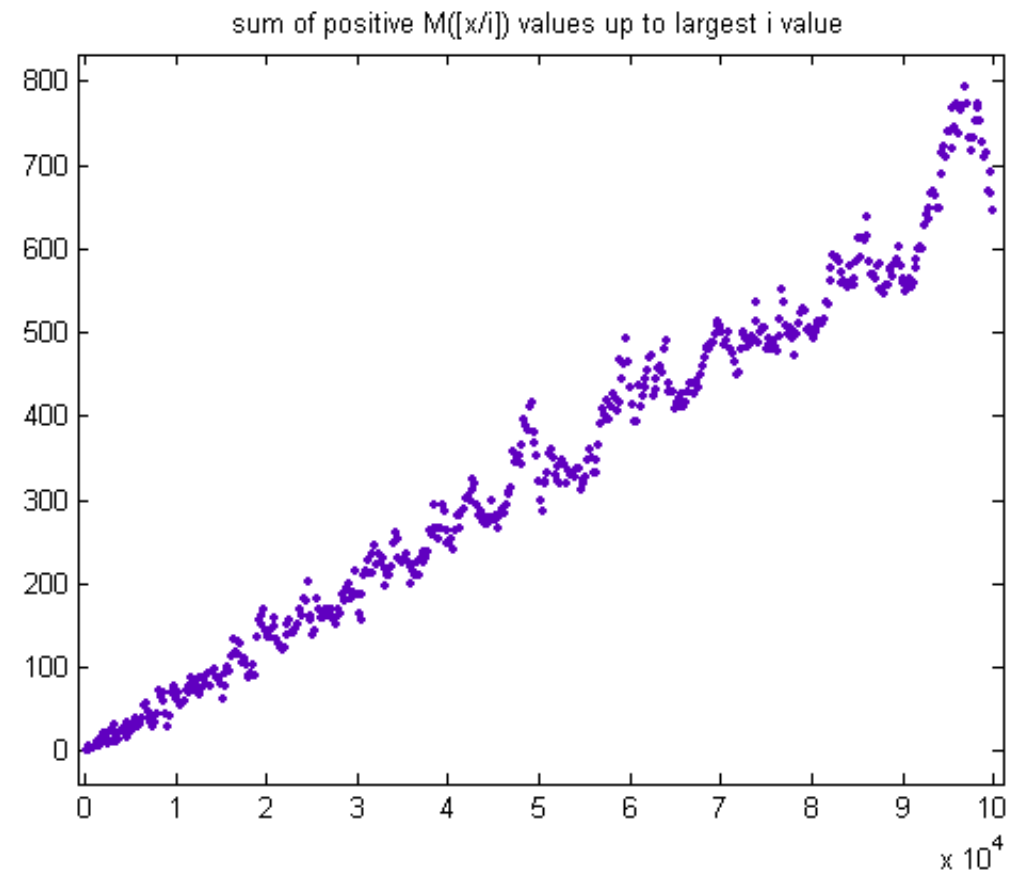

Figure 9: Plot of sum of positive $M(\lfloor x / i\rfloor)$ values up to largest $i$ value

A plot of the number of $M(\lfloor x / i\rfloor)$ values equal to 0 up to the largest $i$ value is given in Figure 10. 


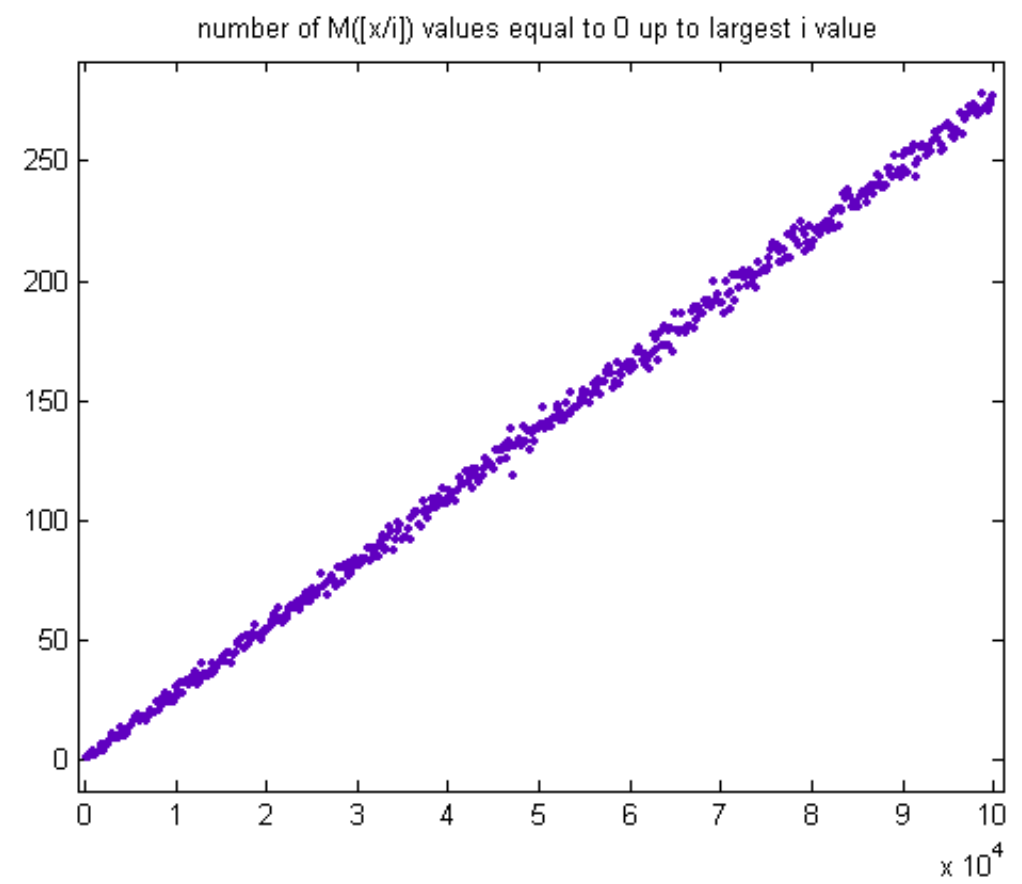

Figure 10: Plot of number of $M(\lfloor x / i\rfloor)$ values equal to 0 up to largest $i$ value

More properties of the sign of the Mertens function are given by Cox [5], et. al. The autocorrelation of the sequence $M(\lfloor x / 1\rfloor), M(\lfloor x / 2\rfloor), M(\lfloor x / 3\rfloor), \ldots$, $M(\lfloor x / x\rfloor)$ can be used to determine a likely upper bound of the absolute value of the Mertens function. See Cox [6], et. al. for details.

\section{The Second Chebyshev Function}

Let $V_{x}$ denote the matrix obtained from $T_{x}$ by element-by-element multiplication of the columns by $\Lambda(j)$. The sum of the sums of the columns is then $\psi(x)$. Let $r_{n}, n=1,2,3, \ldots, x$, denote the sums of the rows. A plot of $r_{n}$ for $n=$ $1,2,3, \ldots, 200$ is given in Figure 11. 


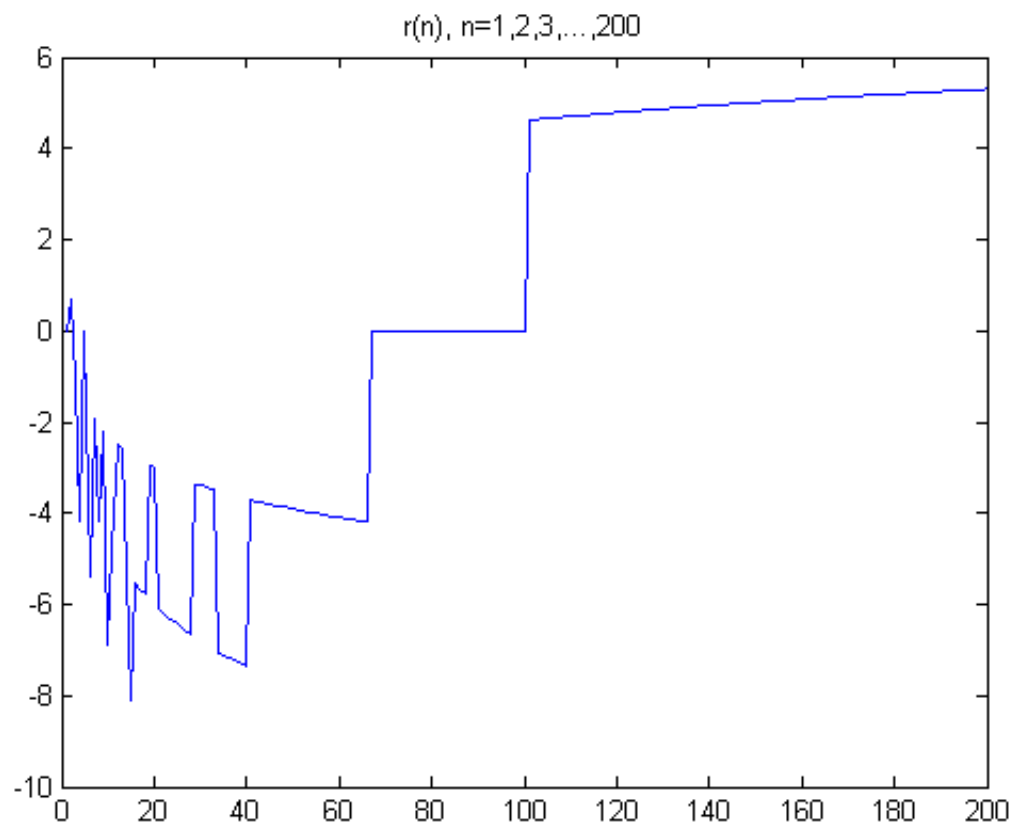

Figure 11: Plot of $r_{n}$ for $n=1,2,3, \ldots 200$

A plot of the values where the first five sums of rows are deleted is given in Figure 12. 


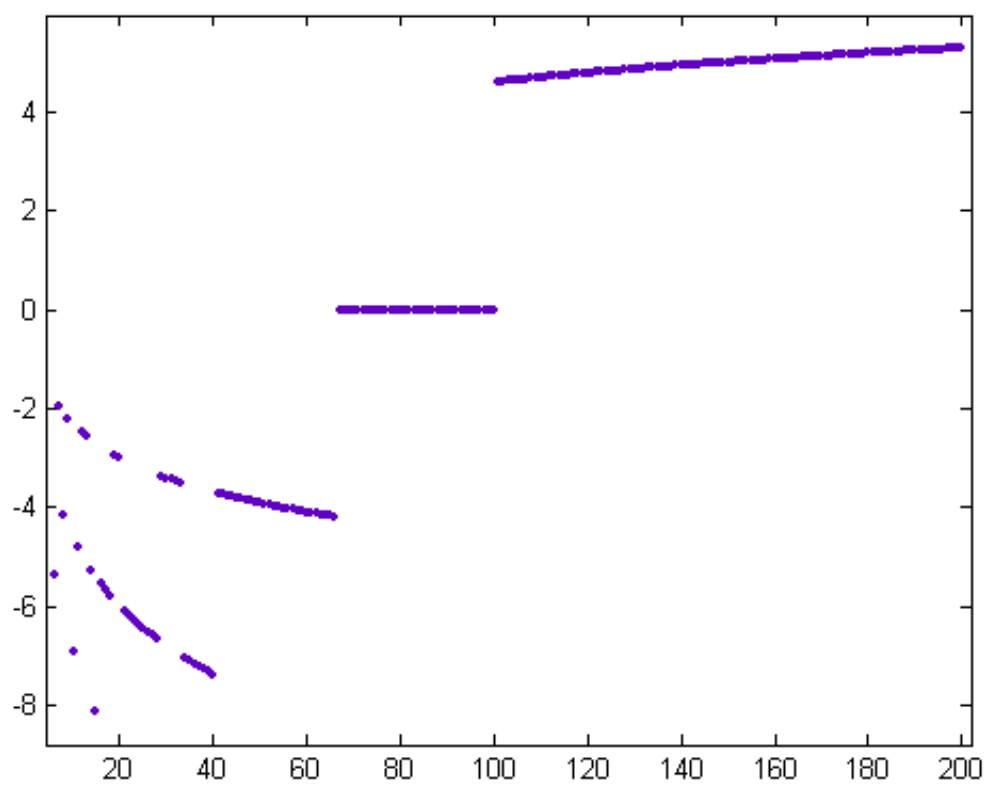

Figure 12: Plot of $r_{n}$ for $n=6,7,8, \ldots 200$

There are three intermittent quadratic curves with negative values.

These plots indicate that $\psi(x)$ equals a series involving the squarefree numbers $1,2,3,5,6,7,10, \ldots$ Each of the "line segments" in the above plot is approximately quadratic. For $x=200$, the $r_{n}$ values from $n=\lfloor x / 2\rfloor+1$ to $\lfloor x / 1\rfloor$ (101, $102,103, \ldots, 200)$ are $\log (101), \log (102), \log (103), \ldots, \log (200)$. A plot of these values is given in Figure 13. 
r(n) values, $n=101,102,103, \ldots, 200$

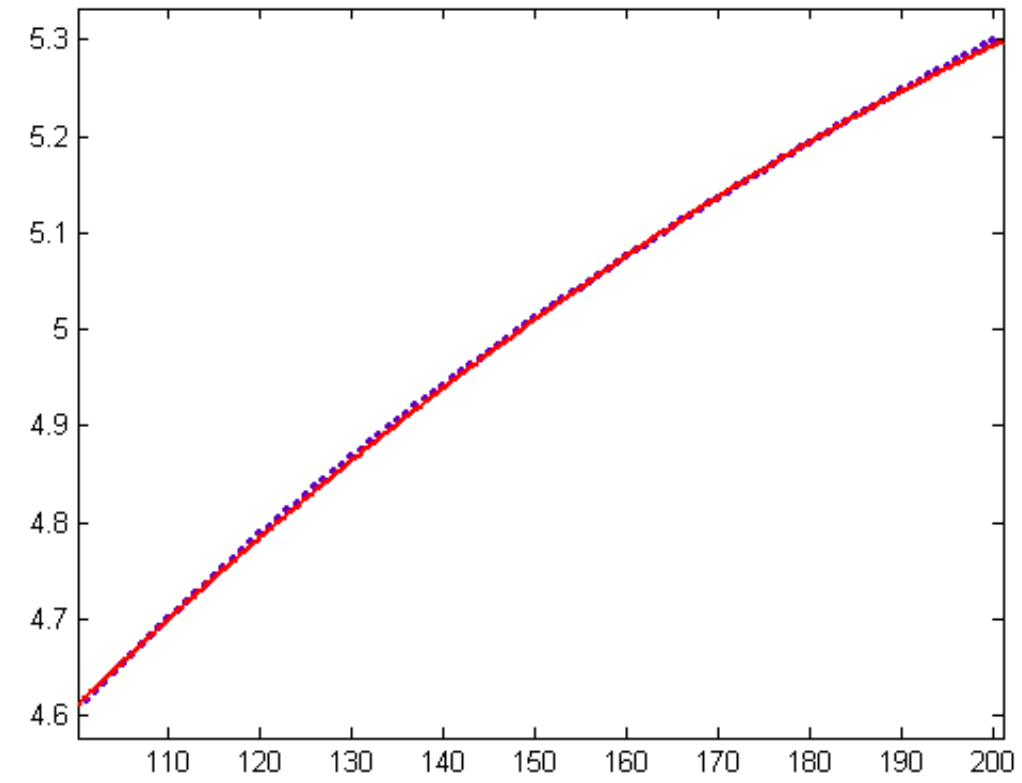

Figure 13: Plot of values from $n=\lfloor x / 2\rfloor+1$ to $\lfloor x / 1\rfloor(101,102,103, \ldots, 200)$

For a quadratic least-squares fit of the data, $p_{1}=-2.319 \cdot 10^{-5}$ with a $95 \%$ confidence interval of $\left(-2.373 \cdot 10^{-5},-2.264 \cdot 10^{-5}\right), p_{2}=0.01378$ with a $95 \%$ confidence interval of $(0.01361,0.01394), p_{3}=3.466$ with a $95 \%$ confidence interval of $(3.454,3.478), \mathrm{SSE}=0.0004042, \mathrm{R}$-squared $=0.9999$, and $\mathrm{RMSE}=0.002041$.

The $r_{n}$ values from $n=\lfloor x / 3\rfloor+1$ to $\lfloor x / 2\rfloor(67,69,70, \ldots, 100)$ are 0 . The $r_{n}$ values from $n=\lfloor x / 5\rfloor+1$ to $\lfloor x / 3\rfloor(41,42,43, \ldots, 66)$ are $-\log (41),-\log (42)$, $-\log (43), \ldots,-\log (66)$. A plot of these values is given in Figure 14 . 


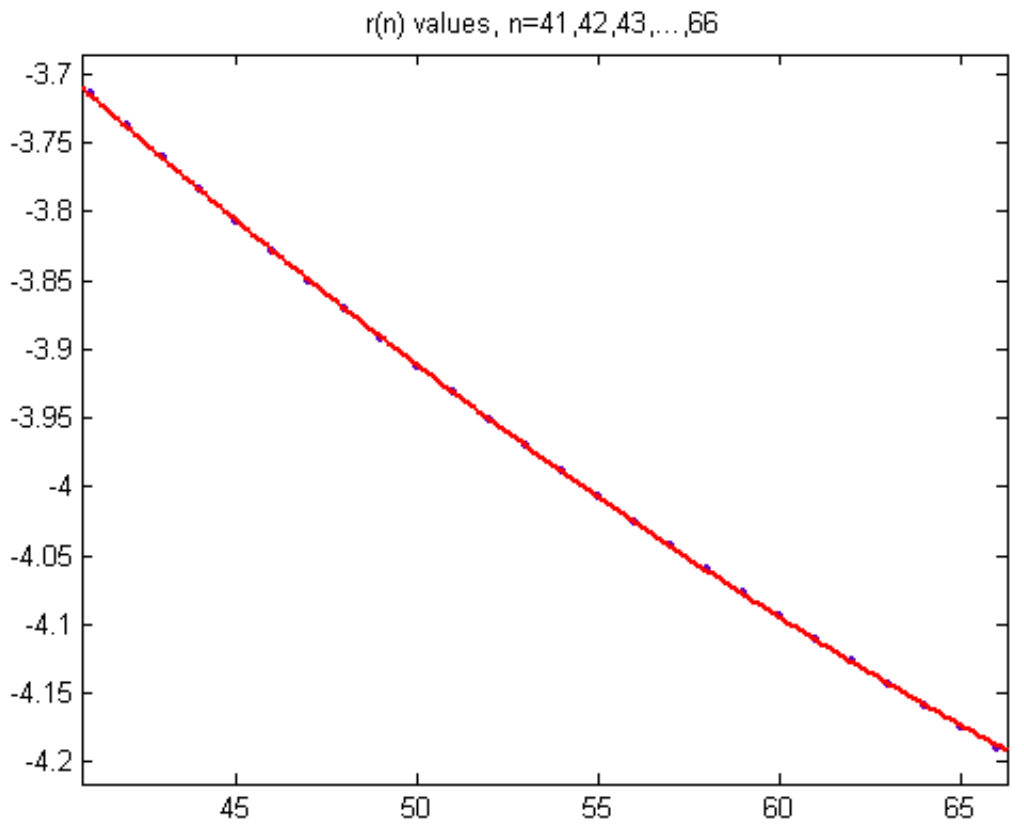

Figure 14: Plot of $r_{n}$ values from $n=\lfloor x / 5\rfloor+1$ to $\lfloor x / 3\rfloor(41,42,43, \ldots, 66)$

For a quadratic least-squares fit of the data, $p_{1}=0.0001795$ with a $95 \%$ confidence interval of $(0.0001728,0.0001857), p_{2}=-0.03809$ with a $95 \%$ confidence interval of $(-0.03878,-0.03741), p_{3}=-0.2 .455$ with a $95 \%$ confidence interval of $(-2.473,-2.437), \mathrm{SSE}=1.454 \cdot 10^{-5}, \mathrm{R}$-squared $=1$, and $\mathrm{RMSE}=0.000795$.

The $r_{n}$ values from $n=\lfloor x / 6\rfloor+1$ to $\lfloor x / 5\rfloor(34,35,36, \ldots, 40)$ are $-2 \log (34)$, $-2 \log (35),-2 \log (36), \ldots,-2 \log (40)$. A plot of these values is given in Figure 15. 


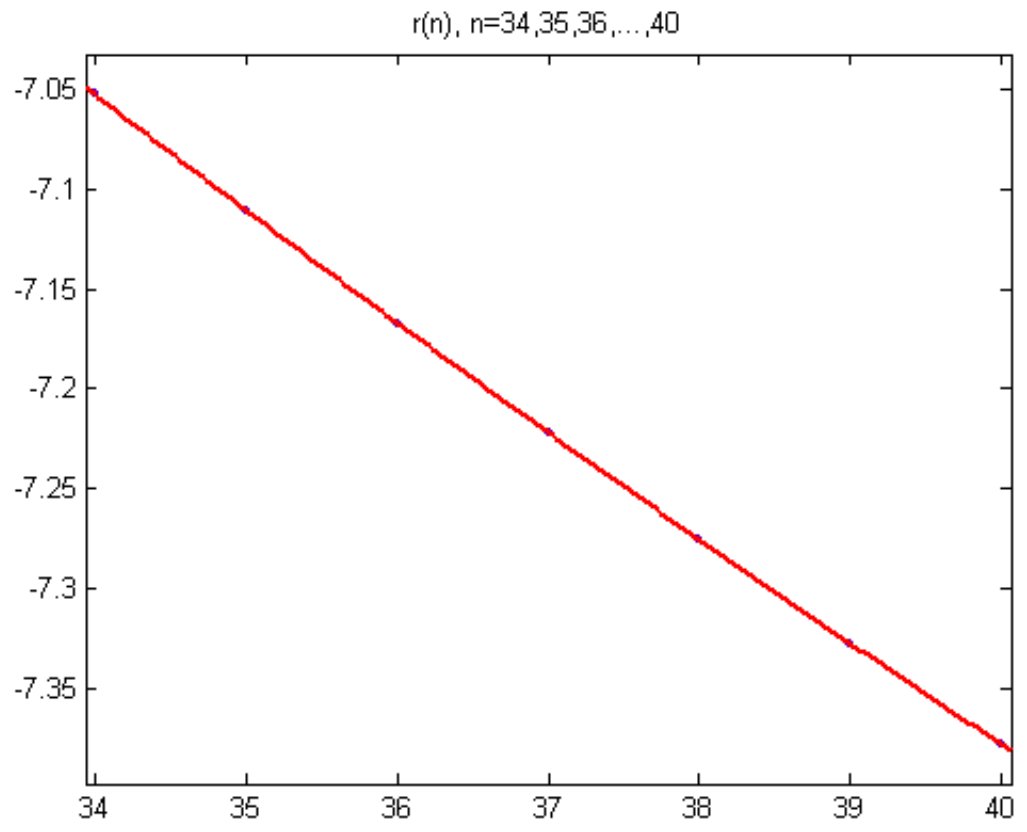

Figure 15: Plot of $r_{n}$ values from $n=\lfloor x / 6\rfloor+1$ to $\lfloor x / 5\rfloor(34,35,36, \ldots, 40)$

For a quadratic least-squares fit of the data, $p_{1}=0.000733$ with a $95 \%$ confidence interval of $(0.0007035,0.0007625), p_{2}=-0.1084$ with a $95 \%$ confidence interval of $(-0.1106,-0.1062), p_{3}=-4.215$ with a $95 \%$ confidence interval of $(-4.255,-4.175), \mathrm{SSE}=3.795 \cdot 10^{-8}, \mathrm{R}$-squared $=1$, and $\mathrm{RMSE}=9.75 \cdot 10^{-5}$.

As with $\Phi(x)$, the process continues with larger squarefree divisors. Again, the multiple for a squarefree number $q$ is $M(q-1)$. A plot of the partial sum of the Chebyshev function corresponding to the squarefree numbers $2,3,5,6,7$, and 10 is given in Figure 16. 


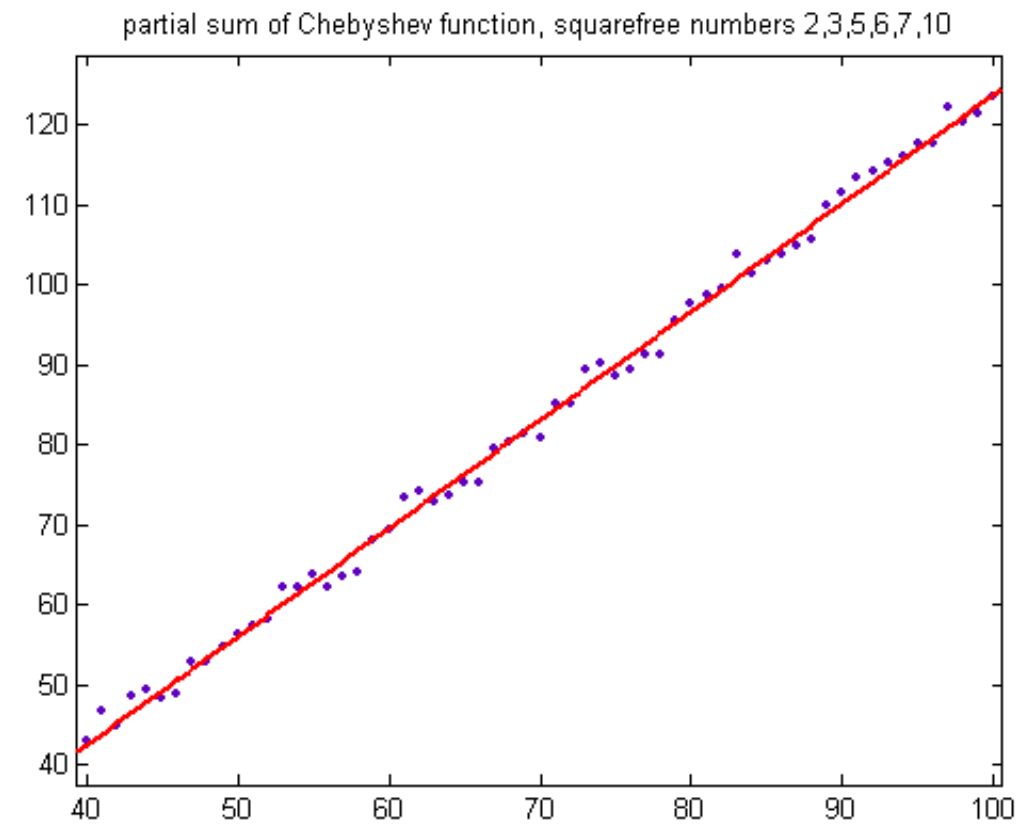

Figure 16: Plot of the partial sum of the Chebyshev function corresponding to the squarefree numbers $2,3,5,6,7$, and 10

For a linear least-squares fit of the data, $p_{1}=1.353$ with a $95 \%$ confidence interval of $(.1331,1.374), p_{2}=-11.53$ with a $95 \%$ confidence interval of $(-13.09$, $-10.02), \mathrm{SSE}=125.3, \mathrm{R}$-squared $=0.9964$, and $\mathrm{RMSE}=1.457$. The slope is greater than 1 and the SSE and RMSE are less than that for $\psi(x)$. A form of the prime number theorem is

(5) $\lim _{x \rightarrow \infty} \frac{\psi(x)}{x}=1$

See Theorem 4.4 of Apostol's book.

For $x=20, \psi(x)=19.26566$. The next $x$ value is not a prime power, so the value does not change. This is effected in the above by the logarithms of the factors of 21 canceling out $-\log (21)-\log (7)-\log (3)=0$. Similarly, the $\operatorname{logarithms}$ of the factors of 22 cancel out $-\log (22)-\log (11)-\log (2)=0$. The next $x$ value is prime. For $x=23, \psi(x)=22.40115$, an increase of $\log (23)$. The next $x$ value is not a prime power. The logarithms of the factors of 24 cancel out $-\log (24)-\log (12)-\log (8)+\log (6)+\log (4)-\log (3)-\log (2)=0$.

\section{References}

[1] T. M. Apostol, Introduction to Analytic Number Theory, Springer, (1976) 
[2] M. Mikolás, Farey series and their connection with the prime number problem 1, Acta Sci. Math. (Szeged) 13 (1949), 93-117

[3] R. M. Redheffer, Eine explizit lösbare Optimierungsaufgabe, Internat. Schriftenreine Numer. Math., 36 (1977)

[4] Cox, Darrell and Ghosh, Sourangshu and Sultanow, Eldar. (2021). Bounds of the Mertens Functions. Advances in Dynamical Systems and Applications. 16. 35-44

[5] Cox, Darrell and Ghosh, Sourangshu and Sultanow, Eldar, Sign of the Mertens Function, Global Journal of Pure and Applied Mathematics, 17(2):201-208 (2021)

[6] Cox, Darrell and Sultanow, Eldar and Ghosh, Sourangshu, The Energy Spectral Density of the Mertens Function, Global Journal of Pure and Applied Mathematics, 17(2):197-199 (2021) 